\title{
openheart Cholesterol induced heart valve inflammation and injury: efficacy of cholesterol lowering treatment
}

\author{
Layan A El-Khatib, ${ }^{1}$ Heather De Feijter-Rupp, ${ }^{2}$ Abed Janoudi, ${ }^{2}$ Levi Fry, ${ }^{2}$ \\ Michael Kehdi, ${ }^{2}$ George S Abela (1) ${ }^{2,3}$
}

To cite: El-Khatib LA, De Feijter-Rupp H, Janoudi A, et al. Cholesterol induced heart valve inflammation and injury: efficacy of cholesterol lowering treatment. Open Heart 2020;7:e001274. doi:10.1136/ openhrt-2020-001274

Received 21 February 2020

Revised 11 May 2020

Accepted 9 June 2020
Check for updates

\section{(c) Author(s) (or their} employer(s)) 2020. Re-use permitted under CC BY-NC. No commercial re-use. See rights and permissions. Published by BMJ.

${ }^{1}$ Internal Medicine, Henry Ford Hospital, Detroit, Michigan, USA 2Department of Medicine, Division of Cardiovascular Medicine, Michigan State University College of Human Medicine, East Lansing, Michigan, USA

${ }^{3}$ Department of Physiology, Division of Pathology, Michigan State University, East Lansing, Michigan, USA

Correspondence to

George S Abela; abela@msu.edu

\section{ABSTRACT}

Background Heart valves often undergo a degenerative process leading to mechanical dysfunction that requires valve replacement. This process has been compared with atherosclerosis because of shared pathology and risk factors. In this study, we aimed to elucidate the role of inflammation triggered by cholesterol infiltration and cholesterol crystals formation causing mechanical and biochemical injury in heart valves.

Methods Human and atherosclerotic rabbit heart valves were evaluated. New Zealand White male rabbits were fed an enriched cholesterol diet alone or with simvastatin and ezetimibe simultaneous or after 6 months of initiating cholesterol diet. Inflammation was measured using Creactive protein (CRP) and RAM 11 of tissue macrophage content. Cholesterol crystal presence and content in valves was evaluated using scanning electron microscopy. Results Cholesterol diet alone induced cholesterol infiltration of valves with associated increased inflammation. Tissue cholesterol, CRP levels and RAM 11 were significantly lower in simvastatin and ezetimibe rabbit groups compared with cholesterol diet alone. However, the treatment was effective only when initiated with a cholesterol diet but not after lipid infiltration in valves. Aortic valve cholesterol content was significantly greater than all other cardiac valves. Extensive amounts of cholesterol crystals were noted in rabbit valves on cholesterol diet and in diseased human valves.

Conclusions Prevention of valve infiltration with cholesterol and reduced inflammation by simvastatin and ezetimibe was effective only when given during the initiation of high cholesterol diet but was not effective when given following infiltration of cholesterol into the valve matrix.

\section{INTRODUCTION}

Heart valves are composed of tissue elements similar to those present in arteries. ${ }^{1}$ They have cells with features related to smooth muscle cells and an organisational structure comparable to the vascular intima. Thus, considerable heart valve pathology seems to overlap with atherosclerotic vascular disease. Inflammation in cardiac valves is known to be a part of rheumatic fever with sequelae that eventually lead to commissural fusion

\section{Key questions}

What is already known about this subject?

- Cardiac valve disease is a common cardiovascular condition that often involves valve sclerosis and calcification. A relationship to atherosclerosis has been proposed but lipid lowering does not seem to alter its course.

What does this study add?

- This study demonstrates that lipid lowering treatment can reduce the inflammation and valve sclerosis if initiated prior to onset of the disease. However, once lipid infiltration sets in, lipid lowering seems to have an insignificant effect. Also, cholesterol crystals present in the valves trigger an inflammatory process similar to atherosclerosis.

How might this impact on clinical practice?

- If used early in the course, lipid lowering may help prevent development of valve sclerosis and dysfunction.

and ultimately calcification. ${ }^{2}$ In the arterial system, inflammation is recognised as integral to atherosclerosis and has been linked to the presence of oxidised cholesterol, cholesterol crystal formation within atherosclerotic plaque and eventual calcification. ${ }^{34}$ In valves, the risk factors leading to valve fibrosis and calcification have been associated with older age, male gender, elevated levels of low density lipoprotein, hypertension, diabetes and genetic predisposition. ${ }^{5}$ These are similar to risk factors associated with atherosclerotic arterial disease. Valve sclerosis and calcification are common causes of valve distortion with dysfunction that results in stenosis and/ or regurgitation often requiring surgical intervention. ${ }^{67}$ Moreover, cardiac valves have certain distinct characteristics that lead to sclerosis based on valve function. ${ }^{8}$

Although retrospective studies in humans had suggested that lipid lowering with statins could result in reduced progression of valve stenosis, ${ }^{9}$ prospective trials did not confirm 
these initial observations. ${ }^{10-12}$ However, in order to better define the underlying pathophysiology that leads to valve injury, we evaluated the cholesterol content, inflammation and presence of crystals in cardiac valves using a combination of simvastatin and ezetimibe in an atherosclerotic rabbit model. Also, we evaluated human cardiac valves for comparison.

\section{METHODS}

Human valves: eleven human cardiac valves including aortic $(n=3)$, mitral $(n=4)$, tricuspid $(n=2)$ and pulmonary $(n=2)$ were obtained either at the time of valve surgery or from postmortem autopsy. These were collected as deidentified samples and taken to the laboratory for examination. Both Michigan State University and Sparrow Hospital institutional review boards approved this protocol (\# 0518-exempt).

Valves from atherosclerotic rabbit model: a total of 124 valves from 32 male New Zealand White rabbits $(2-3 \mathrm{~kg})$ were used in this study; 20 rabbits were made atherosclerotic by balloon de-endothelialisation and feeding a cholesterol enriched diet $(1 \%)$ alternating with normal chow every other month for a period of 6 months. ${ }^{13}$ Group I (Gp I) was atherosclerotic control ( $n=5)$; Gp II atherosclerotic rabbits $(n=10)$ were given a combination of simvastatin $(3 \mathrm{mg} / \mathrm{kg} /$ day $)$ and ezetimibe $(1 \mathrm{mg} / \mathrm{kg} /$ day $)$ during the cholesterol feeding periods; Gp III atherosclerotic rabbits ( $\mathrm{n}=4+1$ dead) were used to simulate pre-existing atherosclerosis by starting simvastatin and ezetimibe combination 6 months following initiation of atherosclerosis; Gp IV was normal control rabbits $(n=12)$ fed normal chow for a period of 6 months. Rabbits were anaesthetised with ketamine $(50 \mathrm{mg} / \mathrm{kg} \mathrm{im})$ and xylazine $(20 \mathrm{mg} / \mathrm{kg} \mathrm{im})$ during balloon de-endothelialization. Buprenorphine $(0.01 \mathrm{mg} / \mathrm{kg} \mathrm{sq})$ was given every 12 hours for 48 hours and antibiotics (enrofloxacin, $10 \mathrm{mg} / \mathrm{kg} \mathrm{sq}$ ) was given once. After euthanasia, rabbit hearts were removed and all four cardiac valves were dissected, removed and processed for biochemical analysis and microscopy.

Serum and valve cholesterol levels: total serum and valve tissue cholesterol concentrations were determined using a kit according to the manufacturer's instructions (Thermo Electron Corp, Louisville, CO).

Serum C-reactive protein (CRP): an ELISA kit from Immunology Consultant Laboratory (Newberg, OR) was used to measure serum CRP at baseline, 6 and 12 months.

Microscopy: light microscopy (LM), scanning electron microscopy (SEM), confocal microscopy (CM) and Keyence 3D microscopy were performed. For LM and SEM, valve segments from all rabbits were fixed overnight in buffered $10 \%$ formalin or $4 \%$ glutaraldehyde, respectively.

$L M$ : fixed tissue segments were serially dehydrated with graded ethanol, embedded in paraffin blocks, and cut in $5 \mu \mathrm{m}$ sections using a microtome. These sections were stained with hematoxylin and eosin for examination under a light microscope (Laborlux12, Leitz, Oberkochen, Germany).

$R A M$ 11: formalin fixed, paraffin embedded, rabbit valve sections were processed with RAM 11 (DAKO, Agilent, Santa Clara, CA), a monoclonal antibody that reacts with a cytoplasmic antigen in the rabbit macrophage. To quantitate macrophage positive areas, tissue sections were scanned in one batch with a slide scanner (Olympus vs 110, Tokyo, Japan) at 20× magnification. Using software (VISIOPHARM, Hoersholm, Denmark) at 10× magnification, images were converted to tagged image file format for analysis in ImageJ V.1.51 k (http:// rsb.info.nih.gov/ij/). The region of interest tool was then used to measure areas of the valve tissue that stained brown with 3,3'-diaminobenzidine, the chromogen for RAM 11. This was then used to calculate the percent of the total valve area stained.

Fluorescence microscopy: tissues were processed as previously described. ${ }^{13}$ Fresh segments of valve tissues were incubated for 4 hours at $37^{\circ} \mathrm{C}$ in Eagle minimum essential medium under $\mathrm{O}_{2}$ and $\mathrm{CO}_{2}$ atmosphere with $10 \mu \mathrm{g} /$ mL Alexa Fluor 594 acetylated-low density lipoprotein (Molecular Probes, Eugene, OR) specific for endothelium. Following incubation, valve tissue was washed with physiological buffered saline (PBS) and fixed with $4 \%$ glutaraldehyde. ${ }^{14}$ The tissue was then counterstained for cholesterol crystals using a green fluorescent dye (cholesteryl Bodipy-C12, Invitrogen, Eugene, OR) at a $1 / 100$ dilution ( $75 \%$ ethanol) in a test tube for $3 \mathrm{~min}^{15}$ Samples were then transferred to a slide incubator chamber filled with PBS for microscopic examination. Unstained tissue samples were also examined for native tissue fluorescence. Fluorescent images were acquired using a Zeiss Pascal LSM microscope (Carl Zeiss, Jena, Germany).

SEM: tissue samples from various valves (3-5 mm long) were dehydrated for 12 hours in a vacuum chamber (Speed Vac SC110, Savant Instruments, Farmingdale, NY) evacuated by a pump (VP110, Franklin Electric, Bluffton, IN). This approach was used because standard methods using ethanol dissolve cholesterol crystals. ${ }^{16}$ These tissue segments were then mounted on stubs and gold coated in a sputter coater (EMSCOPE SC500; Emscope, Ashford, UK). The valve surfaces were then examined using a Jeol SEM (model JSM-6300F, Jeol Ltd, Tokyo, Japan).

Transmission electron microscopy (TEM): in order to perform TEM on tissues while avoiding solvent agents (ie, uranyl acetate) for cholesterol crystals, we used a fresh frozen approach. Although this reduces the surrounding tissue resolution, it preserves intact cholesterol crystals. Tissues were immersed in a saturated sucrose solution overnight and then underwent cryosectioning. ${ }^{17}$ These were stained with osmium tetroxide. Ultrathin sections were then examined using a Jeol 100 CX electron microscope.

Keyence 3D microscopy: fresh tissue samples were placed under a 3D Keyence VHX-6000 digital microscope 

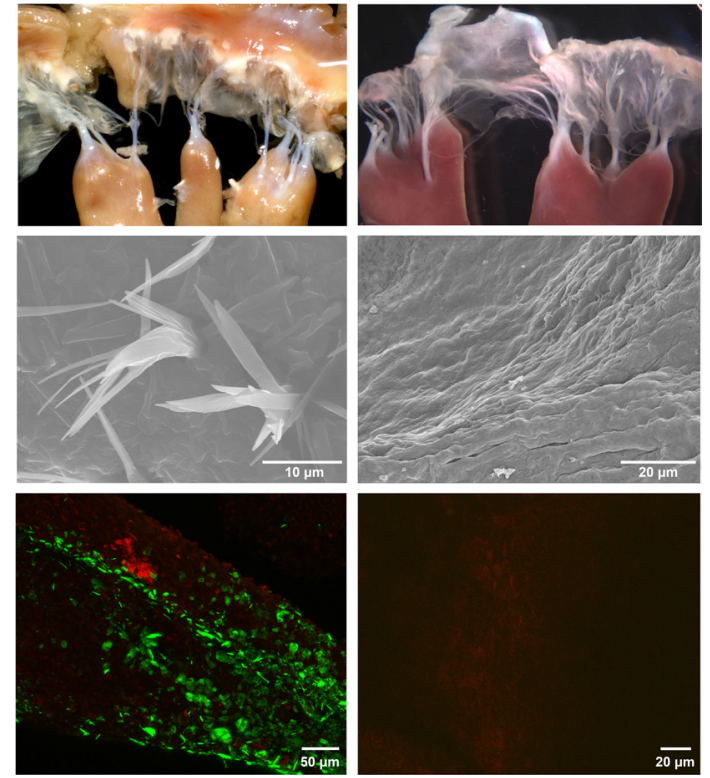

Figure 1 (Left) Gross valve images, scanning electron micrographs and fluorescence microscopy of atherosclerotic rabbit mitral valve demonstrating discoloured and thickened mitral valve with cholesterol crystals perforating the valve surfaces. (Right) Gross valve images, scanning electron micrographs and fluorescence microscopy of normal valve from non-atherosclerotic rabbit without evidence of cholesterol crystals.

(Keyence Corp., Osaka, Japan) and images of crystals obtained using a VH-Z500T high-resolution zoom lens.

\section{Patient and public involvement}

Patients and the public were not involved in this research.

\section{Statistical analysis}

Data are reported as mean \pm SD. Valve cholesterol content, serum cholesterol and serum CRP were analysed by analysis of variance. Percent area of RAM 11 stained macrophages in atherosclerotic rabbit valves, rabbits on high cholesterol diet with simvastatin and ezetimibe and normal controls were compared using Kruskal-Wallis non-parametric test at 0.05 significance level.

\section{RESULTS}

Gross examination: evaluation of cardiac valves by gross examination revealed that rabbits fed a high cholesterol diet had yellow-tinged discoloration and appeared thickened compared with valves from normal control rabbits (figure 1). Human aortic and mitral valves appeared distorted with roughened surfaces.

Serum cholesterol levels: serum cholesterol in the four groups is presented in figure 2. After 2 weeks of cholesterol feeding, serum cholesterol levels increased significantly in Gps I and III but not in Gp II $(\mathrm{p}<0.001)$. This pattern was sustained throughout the first 9 months of the study. However, by month 12, serum cholesterol in Gp III rabbits had also decreased significantly and was similar to Gp II.

Valve cholesterol content: cholesterol tissue content was significantly lower with the simvastatin and ezetimibe combination initiated with cholesterol diet (Gp II) compared with Gps I and III (figure 3). When simvastatin and ezetimibe were initiated 6 months after feeding with high cholesterol diet (Gp III), cholesterol levels in the aortic, mitral and pulmonary valves were not significantly different from the untreated atherosclerotic group (Gp I). Within the untreated atherosclerotic rabbits (Gp I), cholesterol content was significantly higher for the aortic valve compared with all other cardiac valves $(p<0.001)$ (figure 4).

Serum inflammation: CRP levels were similar in Gps I, II and III at baseline prior to initiation of cholesterol diet. At 6 months, CRP levels in Gp II were significantly lower than Gp I $(2720 \pm 2193 \mathrm{ng} / \mathrm{mL}$ vs $10519 \pm 10947 \mathrm{ng} /$ $\mathrm{mL}, \mathrm{p}=0.03)$. At 12 months, both simvastatin plus ezetimibe groups (Gps II and III) were significantly lower

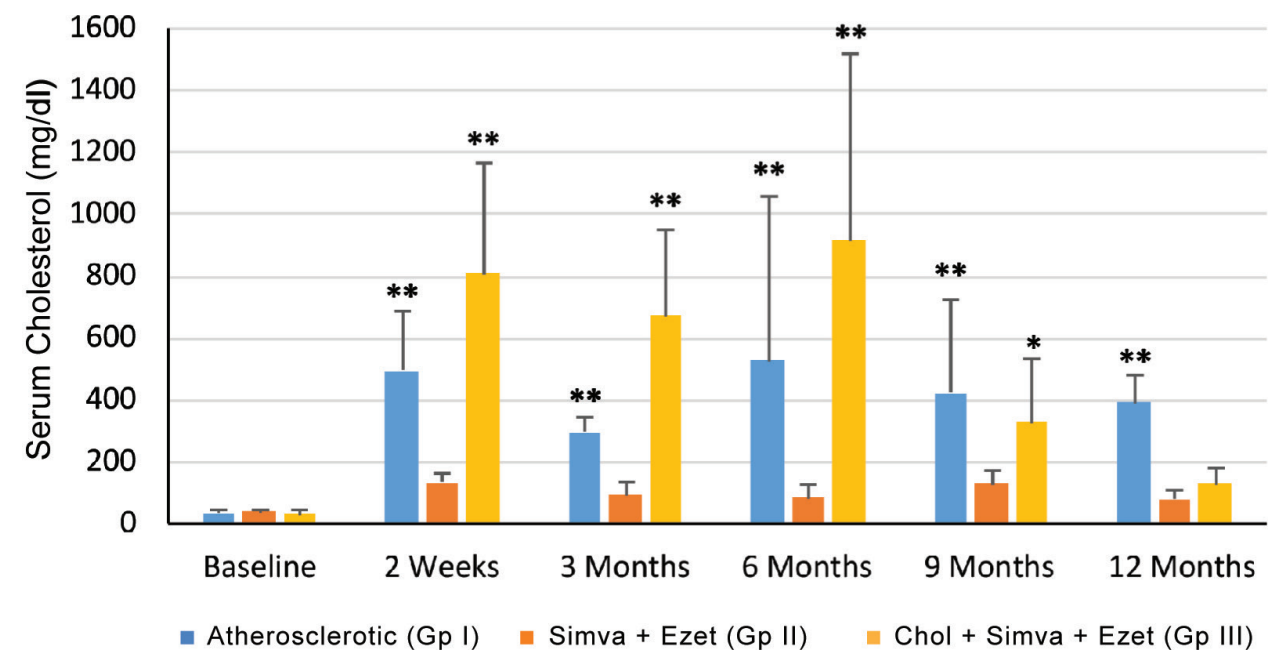

Figure 2 Cholesterol levels in rabbit serum. Chol, cholesterol diet only (Gp I); Simva+Ezet, treatment with simvastatin and ezetimibe started concurrently with cholesterol diet. (Gp II); Chol-Simva+Ezet, treatment with simvastatin and ezetimibe initiated 6 months after starting the cholesterol diet (Gp III). ${ }^{\star *} p<0.001 ;{ }^{*} p<0.05$. 


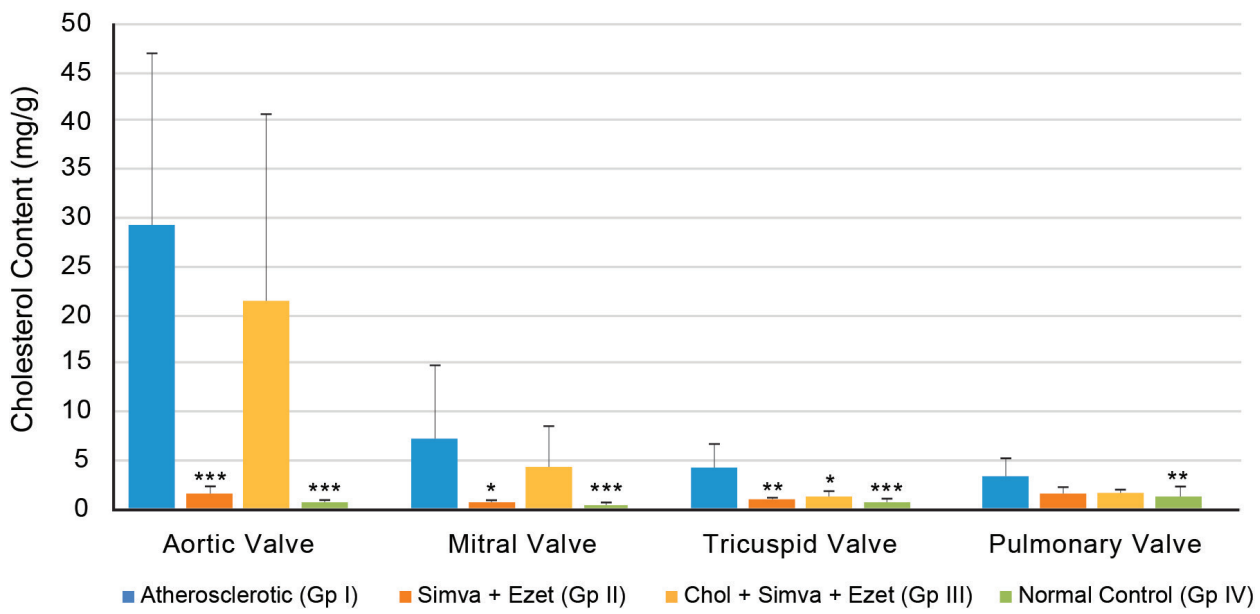

Figure 3 Cholesterol content of rabbit aortic, mitral, tricuspid and pulmonary valves. High cholesterol diet only (Gp I); simvastatin and ezetemide (Simva+Ezet), treatment with Simva+Ezet started concurrently with cholesterol diet (GP II); (Cholesterol-Simva+Ezet)-Chol+Simva+Ezet, treatment with Simva+Ezet initiated 6 months after the initiation of the cholesterol diet (Gp III), and normal control (Gp IV). ${ }^{* \star *} p<0.001 ;{ }^{* *} p<0.01 ;{ }^{*} p<0.05$.

than Gp I $(1256 \pm 650 \mathrm{ng} / \mathrm{mL}$ and $1089 \pm 263 \mathrm{ng} / \mathrm{mL}$ vs 40 $910 \pm 39585 \mathrm{ng} / \mathrm{mL} \mathrm{p}=0.0001)$.

Cardiac valve inflammation (table 1 ): for all valves, inflammation as indicated by RAM 11 staining was significantly reduced by simvastatin and ezetimibe treatment and was similar to normal control.

\section{Microscopy}

RAM 11 staining revealed a significantly greater amount of macrophages in the valve matrix of Gp I atherosclerotic rabbits compared with normal controls (Gp IV) (figure 5).

Dual fluorescent imaging by CM demonstrated extensive cholesterol crystals present over the intimal surface of the human valves similar to rabbit valves (figures 1 and $6)$.

SEM, TEM and 3D Keyence of the valves demonstrated extensive cholesterol crystals protruding from the valve surfaces of both human and rabbit valves (figures 6 and 7 ). Some cholesterol crystals were found buried in the valve tissues of normal controls while cholesterol crystals in the atherosclerotic-treated rabbits had more complex

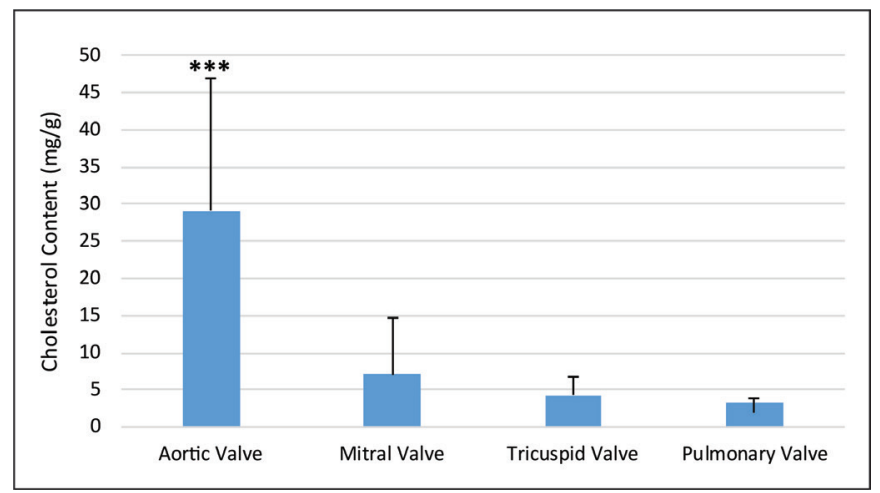

Figure 4 Valve cholesterol content in high cholesterol-fed atherosclerotic rabbits without simvastatin and ezetemide treatment (Gp l) demonstrates aortic valve to have the highest cholesterol content. ${ }^{* *} p<0.001$. formations that protruded through the intimal surface. Atherosclerotic rabbits that were on simvastatin and ezetimibe (Gps II and III) had evidence of dissolving crystals exhibited by loss of sharp edges and erosion of their central matrix (figure 7). However, not enough specimens were available to detect significant crystal density differences between the groups.

\section{DISCUSSION}

Our study demonstrated that high cholesterol diet results in uptake of cholesterol into cardiac valves and triggers inflammation as evidenced by extensive macrophage infiltration into the valve matrix with an associated elevation in serum CRP levels. Moreover, the presence of cholesterol crystals in the atherosclerotic arteries is a known cause of inflammation. ${ }^{13} 18$ Although occasional presence of cholesterol crystals in cardiac valves had been previously reported in the valve matrix using $\mathrm{LM},{ }^{19}$ the extensiveness of cholesterol crystals lining the valve surfaces was not previously appreciated because tissue processed using ethanol dehydration dissolves cholesterol crystals. Our data also demonstrated presence of cholesterol crystals on diseased human valves as we have seen for rabbit valves on high cholesterol diet. By utilising SEM, TEM and Keyence microscopy without tissue ethanol dehydration, we were able to detect the extent of cholesterol crystals on valve surfaces and their intimal damage. ${ }^{16}$ These findings help elucidate the underlying mechanism of inflammation leading to valve injury. ${ }^{20}$

Although cholesterol deposits and cholesterol crystals are a hallmark of atherosclerosis, we have now demonstrated that in the presence of hypercholesterolemia, these are also very common features in valve tissue. In atherosclerosis, cholesterol crystals have been found to trigger an inflammatory response in the plaque as well as systemically. ${ }^{13} 18$ In our study, we find a similar effect as was evidenced by dense macrophage infiltration 


\begin{tabular}{lllll}
\hline Table 1 & Cardiac valve inflammation by percent RAM 11 area staining of & & \\
\hline Treatment group & $\begin{array}{l}\text { Aortic } \\
\text { (\% area) }\end{array}$ & $\begin{array}{l}\text { Mitral } \\
\text { (\% area) }\end{array}$ & $\begin{array}{l}\text { Pulmonary } \\
\text { (\% area) }\end{array}$ & $\begin{array}{l}\text { Tricuspid } \\
\text { (\% area) }\end{array}$ \\
\hline Gp I: Atherosclerotic $(n=5)$ & 29.0 & 35.8 & 28.1 & 7.3 \\
Gp II: Simvastatin+Ezetimibe $\left(n=8^{\star}\right)$ & 0.1 & 0.0 & 0.0 & 0.0 \\
Gp IV: Normal Control $(n=12)$ & 0.1 & 0.0 & 0.0 & 0.0 \\
P value & 0.002 & 0.02 & 0.002 & 0.002 \\
\hline
\end{tabular}

${ }^{*}$ In Gp II, not all valves were available for RAM11, two were used to measure cholesterol content.

demonstrated by RAM 11 staining as well as serum CRP elevation. In the atherosclerotic rabbit model, we had previously demonstrated a strong association between elevated serum inflammatory biomarkers and RAM 11 with cholesterol crystal density in arterial tissues during atherogenesis. ${ }^{13}$ In the current study, we describe a similar finding with RAM 11 in valve tissue. The atherosclerotic rabbit model has been well established and validated as an effective model to study the effects of hyperlipidemia. ${ }^{21}{ }^{22}$ Thus, the evaluation of the cardiac valves in this atherosclerotic model was helpful to examine the effect of lipid lowering with simvastatin and ezetimibe.

Another important observation from our study is that once the valves have been infiltrated by cholesterol and especially when cholesterol crystallises, it becomes very difficult to reverse this process. Specifically, the crystalline state is relatively inert and not easily degradable except by high-density lipoprotein cholesterol (HDLc) which endogenously is the only known biological molecule to solubilise cholesterol crystals. ${ }^{323}$ Especially, when cholesterol crystals are buried deep in the tissue matrix, as has been demonstrated by optical coherence tomography, it is difficult for the HDLc to reach and mobilise the crystals. ${ }^{24}$ In our study, the use of simvastatin and ezetimibe was not effective in lowering the amount of cholesterol in
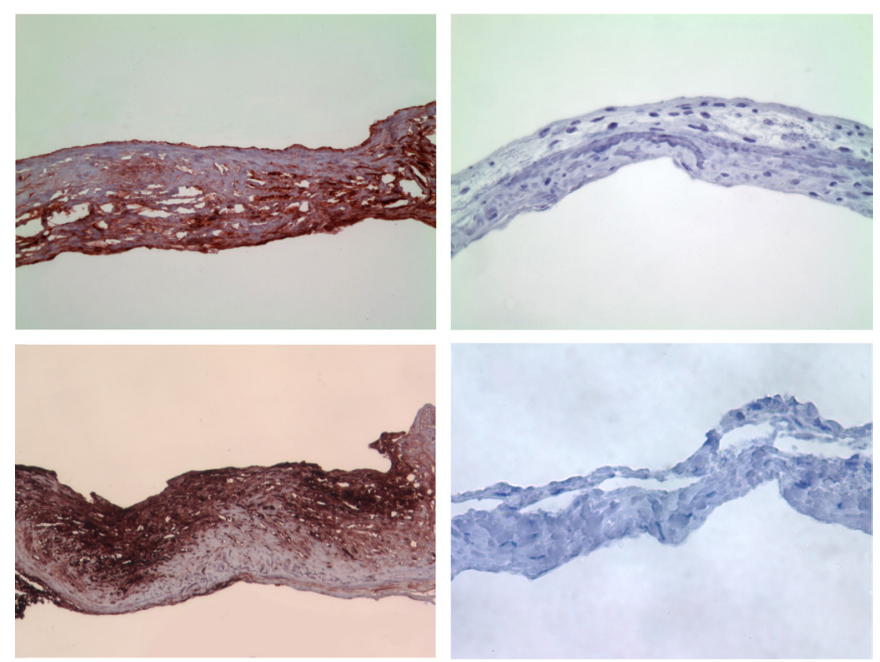

Figure 5 (Left panel) RAM 11 stained aortic (top 200x) and mitral (bottom 200x) valves demonstrating heavy brown staining consistent with macrophage uptake (Gp I) compared with the normal control (right panel) aortic (top 400x) and mitral (bottom 200x) valves (Gp IV) (right panel). the valve once cholesterol had crystallised. This is consistent with the findings from the Simvastatin and Ezetimibe in Aortic Stenosis trial where the use of simvastatin and ezetimibe in patients with aortic stenosis was not found to be effective in either preventing progression or reversing aortic stenosis. $^{10-12}$

A noteworthy finding in our study was the presence of significantly higher cholesterol content of the aortic
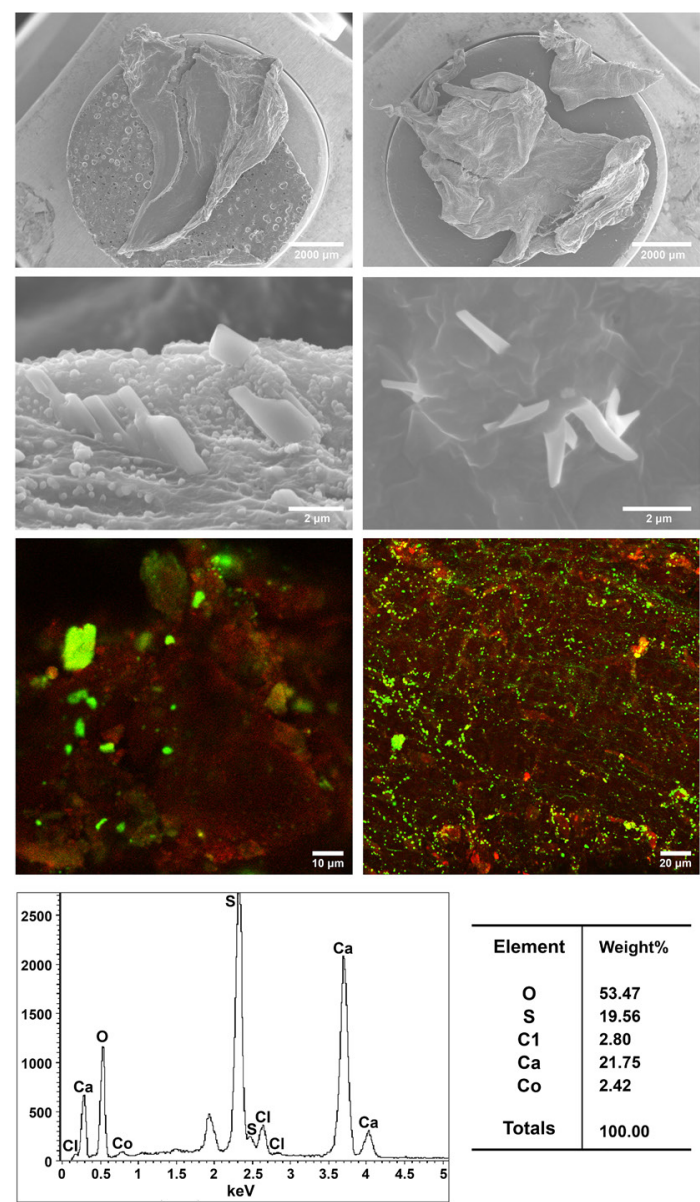

Figure 6 (Top panel) Scanning electron micrographs of human aortic (left) and mitral (right) valves with cholesterol crystals that appear to be emerging from the surface of the valves. Fluorescence microscopy demonstrates cholesterol crystals at the valve surface in fresh unprocessed tissue (bodipy staining cholesterol crystals green and Ac-LDL counter stained endothelium red). (Bottom) Crystallography demonstrates the presence of three calcium peaks on the aortic valve. 


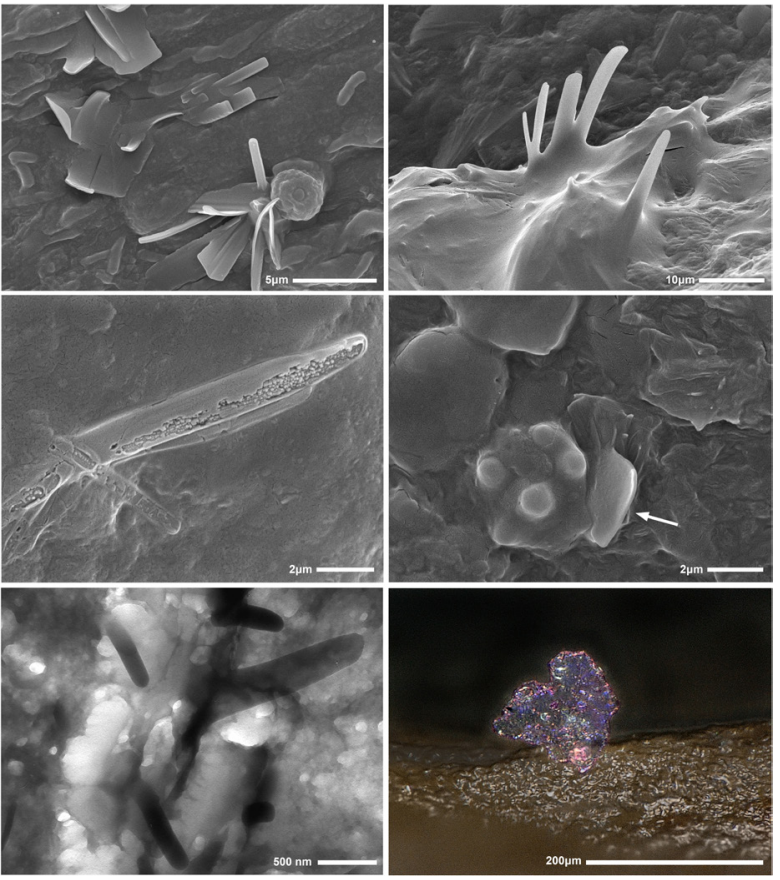

Figure 7 (Top two panels) Scanning electron micrographs of cholesterol crystals emerging from the surface of valves in atherosclerotic rabbit (Gp I). Middle panel demonstrates a dissolving cholesterol crystal with moth eaten appearance from Gp II on simvastatin and ezetimibe. Macrophage is noted on valve next to a cholesterol crystal (arrow). Bottom panel demonstrates cholesterol crystals emerging to the surface by transmission electron and 3D Keyence microscopy, respectively.

valve compared with other valves. Specifically, in elderly patients, the aortic valve is usually the most frequently affected by calcification and stenosis. ${ }^{25}$ It has been demonstrated that high cholesterol saturation has been found to trigger cholesterol crystal formation. ${ }^{26}$ Moreover, studies have implicated cholesterol crystals in the initiation of calcification by serving as a nidus for calcium deposition. ${ }^{27} 28$ This has been demonstrated in both bench top studies as well as ex vivo models. ${ }^{28}{ }^{29}$ Also, studies have demonstrated that cholesterol crystal formation contributes to both mechanical injury as well as inflammation in atherosclerotic plaque. ${ }^{131830}$ Since the aortic valve accumulates the greatest amount of cholesterol, this suggests that this could be a cause of its frequent calcification. The exposure of the aortic valve to high pressure gradients compared with other valves may be the reason for the greater amount of cholesterol deposition and inflammation. ${ }^{25}{ }^{31}$ In contradistinction, the right heart valves, tricuspid and pulmonary, that are exposed to low pressures had the least amount of cholesterol accumulation and these valves have the least amount of calcification in humans as well.

Another implication of our findings relates to the risk for development of bacterial endocarditis. It has been previously demonstrated that various bacteria selectively attach to cholesterol crystals. Also, in the presence of human carotid arterial plaques with cholesterol crystals, there was enhanced bacterial growth (Staphylococcus aureus) when compared with normal carotid arteries. ${ }^{32}$ The bacteria in that study were found to be degrading the cholesterol crystals, possibly using them as an energy source. Such a mechanism has been demonstrated with Mycobacterium tuberculosis. ${ }^{33}$

In summary, the presence of cholesterol and cholesterol crystal deposits in valve tissue can trigger inflammation which in turn can lead to valve calcification. Although cholesterol lowering treatment with simvastatin and ezetimibe significantly lowered valve cholesterol content when initiated as a preventive treatment, it was not effective when treatment was initiated after cholesterol deposition in the valve had occurred. This validates the observation made during clinical trials using this combination of lipid lowering that failed to prevent continued progression of aortic valve stenosis. These observations may be related to the difficulty for the human body to mobilise cholesterol crystals deposited deeply within the valve matrix. However, our study suggests that early treatment with lipid lowering may be considered for patients who are at high risk for cholesterol tissue deposition (ie, familial hypercholesterolemia). Further clinical studies are needed to investigate approaches to prevent cholesterol infiltration into cardiac valves.

\section{Limitations}

Number of valves were small in both the human and the rabbit groups because it was not feasible to obtain sufficient valve tissue for both cholesterol content measurements and histologic analysis with SEM. Also, some of the fluctuation in serum cholesterol levels at 3 and 6 months in figure 2 may be related to the cycling of the cholesterol enriched diet and normal chow but that did not influence the overall trends.

Acknowledgements The authors thank Ruiping Huang, PhD Department of Medicine/Cardiology Research Laboratory for conducted biochemical assays; Amy Porter, HT, QIHC, Investigative Histopathology Laboratory for tissue histology and immunohistochemistry; Abby Vanderberg, BSc and Melinda Frame, PhD Center for Advanced Microscopy for SEM and confocal microscopy, Michigan State University, East Lansing, Michigan.

Contributors LAE-K contributed to writing the manuscript and data analysis. HD-R collected data and processed tissue for SEM. AJ conducted data analysis and helped in writing the manuscript. LF and MK reviewed the manuscript and provided critique. GA wrote the manuscript and provided data interpretation.

Funding Department of Medicine and College of Human Medicine, Michigan State University, East Lansing, Michigan, Merck-Schering/Plough, Kenilworth, NJ. and Edward Sparrow Hospital, Lansing, Ml.

Competing interests GA is a speaker for Amgen and Kowa Pharmaceutical. He is a former recipient of grant from Merck.

Patient consent for publication Not required.

Ethics approval This protocol was approved by Michigan State University's Animal Care and Use Committee following National Institute of Health guidelines (Institutional Animal Care and Use Committee \# 03/18-034-01).

Provenance and peer review Not commissioned; externally peer reviewed.

Data availability statement All data relevant to the study are included in the article or uploaded as supplementary information. All data is presented and anlaysed in the manuscript. 
Open access This is an open access article distributed in accordance with the Creative Commons Attribution Non Commercial (CC BY-NC 4.0) license, which permits others to distribute, remix, adapt, build upon this work non-commercially, and license their derivative works on different terms, provided the original work is properly cited, appropriate credit is given, any changes made indicated, and the use is non-commercial. See: http://creativecommons.org/licenses/by-nc/4.0/.

ORCID iD

George S Abela http://orcid.org/0000-0002-8201-956X

\section{REFERENCES}

1 Filip DA, Radu A, Simionescu M. Interstitial cells of the heart valves possess characteristics similar to smooth muscle cells. Circ Res 1986;59:310-20.

2 Carapetis JR, Beaton A, Cunningham MW, et al. Acute rheumatic fever and rheumatic heart disease. Nat Rev Dis Primers 2016;2:15084.

3 Janoudi A, Shamoun FE, Kalavakunta JK, et al. Cholesterol crystal induced arterial inflammation and destabilization of atherosclerotic plaque. Eur Heart J 2016;37:1959-67.

4 Libby P. Inflammation in atherosclerosis. Arterioscler Thromb Vasc Biol 2012;32:2045-51.

5 Stewart BF, Siscovick D, Lind BK, et al. Clinical factors associated with calcific aortic valve disease. Cardiovascular Health Study. J Am Coll Cardiol 1997:29:630-4

6 Sathyamurthy I, Alex S. Calcific aortic valve disease: is it another face of atherosclerosis? Indian Heart J 2015;67:503-6.

7 Abramowitz Y, Jilaihawi H, Chakravarty T, et al. Mitral annulus calcification.. J Am Coll Cardiol 2015;66:1934-41.

8 Ortlepp JR, Schmitz F, Mevissen V, et al. The amount of calciumdeficient hexagonal hydroxyapatite in aortic valves is influenced by gender and associated with genetic polymorphisms in patients with severe calcific aortic stenosis. Eur Heart J 2004;25:514-22.

9 Rosenhek R, Rader F, Loho N, et al. Statins but not angiotensinconverting enzyme inhibitors delay progression of aortic stenosis. Circulation 2004:110:1291-5.

10 Rossebø AB, Pedersen TR, Boman K, et al. Intensive lipid lowering with simvastatin and ezetimibe in aortic stenosis. N Engl J Med 2008;359:1343-56.

11 Cowell SJ, Newby DE, Prescott RJ, et al. Scottish aortic stenosis and lipid lowering trial, impact on regression (SALTIRE) Investigators. A randomized trial of intensive lipid-lowering therapy in calcific aortic stenosis. N Engl J Med 2005;352:2389-97.

12 Chan KL, Teo K, Dumesnil JG, et al. Effect of lipid lowering with rosuvastatin on progression of aortic stenosis: results of the aortic stenosis progression observation: measuring effects of rosuvastatin (ASTRONOMER) trial. Circulation 2010;121:306-14.

13 Patel R, Janoudi A, Vedre A, et al. Plaque rupture and thrombosis are reduced by lowering cholesterol levels and crystallization with ezetimibe and are correlated with fluorodeoxyglucose positron emission tomography. Arterioscler Thromb Vasc Biol 2011;31:2007-14.

14 Voyta JC, Via DP, Butterfield CE, et al. Identification and isolation of endothelial cells based on their increased uptake of acetylated-low density lipoprotein. J Cell Biol 1984;99:2034-40.

15 Abela GS, Aziz K, Vedre A, et al. Effect of cholesterol crystals on plaques and intima in arteries of patients with acute coronary and cerebrovascular syndromes. Am J Cardiol 2009;103:959-68.
16 Nasiri M, Janoudi A, Vanderberg A, et al. Role of cholesterol crystals in atherosclerosis is unmasked by altering tissue preparation methods. Microsc Res Tech 2015;78:969-74.

17 Webster P, Webster A. Cryosectioning fixed and cryoprotected biological materials. In: Kuo J II, ed. Electron microscopy: methods and protocols. Totowa, NJ: Humana Press, 2007: 257-89.

18 Düewell P, Kono H, Rayner KJ, et al. NIrp3 inflammasomes are required for atherogenesis and activated by cholesterol crystals. Nature 2010;464:1357-61.

19 Kuusisto J, Räsänen K, Särkioja T, et al. Atherosclerosis-like lesions of the aortic valve are common in adults of all ages: a necropsy study. Heart 2005;91:576-82.

20 Abela GS. Cholesterol crystals piercing the arterial plaque and intima trigger local and systemic inflammation. J Clin Lipidol 2010;4:156-64.

21 Phinikaridou A, Hallock KJ, Qiao Y, et al. A robust rabbit model of human atherosclerosis and atherothrombosis. $J$ Lipid Res 2009;50:787-97.

22 Abela OG, Ahsan $\mathrm{CH}$, Alreefi $\mathrm{F}$, et al. Plaque rupture and thrombosis: the value of the atherosclerotic rabbit model in defining the mechanism. Curr Atheroscler Rep 2016;18:29.

23 Abdulla $\mathrm{YH}$, Adams $\mathrm{CW}$. The action of human high density lipoprotein on cholesterol crystals. Part 2. Biochemical observations. Atherosclerosis 1978;31:473-80.

24 Nishimura S, Ehara S, Hasegawa T, et al. Cholesterol crystal as a new feature of coronary vulnerable plaques: an optical coherence tomography study. J Cardiol 2017;69:2017:253-9.

25 Freeman RV, Otto CM. Spectrum of calcific aortic valve disease: pathogenesis, disease progression, and treatment strategies. Circulation 2005; 111:3316-26.

26 Vedre A, Pathak DR, Crimp M, et al. Physical factors that trigger cholesterol crystallization leading to plaque rupture. Atherosclerosis 2009;203:89-96.

27 McCullough PA, Chinnaiyan KM, Agrawal V, et al. Amplification of atherosclerotic calcification and Mönckeberg's sclerosis: a spectrum of the same disease process. Adv Chronic Kidney Dis 2008;15:396-412.

28 Dorozhkina El, Dorozhkin SV. In vitro crystallization of carbonateapatite on cholesterol from a modified simulated body fluid. Colloids Surf A Physicochem Eng Asp 2003;223:231-7.

29 Laird DF, Mucalo MR, Yokogawa Y. Growth of calcium hydroxyapatite (Ca-HAp) on cholesterol and cholestanol crystals from a simulated body fluid: a possible insight into the pathological calcifications associated with atherosclerosis. J Colloid Interface Sci 2006;295:348-63.

30 Al-Handawi MB, Commins P, Karothu DP, et al. Mechanical and crystallographic analysis of cholesterol crystals puncturing biological membranes. Chemistry 2018;24:11493-7.

31 Berkelmans GFN, van der Graaf Y, Dorresteijn JAN, et al. Decline in risk of recurrent cardiovascular events in the period 1996 to 2014 partly explained by better treatment of risk factors and less subclinical atherosclerosis. Int J Cardiol 2018;251:96-102.

32 Boumegouas M, Gardiner J, Janoudi A, et al. Abstract: human atherosclerotic carotid artery plaques with cholesterol crystals enhance bacterial growth: risk for plaque destabilization. $J$ Clinc Lipdol 2018;12.

33 Miner MD, Chang JC, Pandey AK, et al. Role of cholesterol in Mycobacterium tuberculosis infection. Indian J Exp Biol 2009;47:407-11. 